\title{
Dynamic Model-Driven Quantitative and Visual Evaluation of the Aortic Valve from 4D $\mathrm{CT}^{\star}$
}

\author{
Razvan Ioan Ionasec ${ }^{1,2, \star \star}$, Bogdan Georgescu $^{1}$, Eva Gassner $^{3}$, Sebastian Vogt ${ }^{4}$, \\ Oliver Kutter ${ }^{2}$, Michael Scheuering ${ }^{4}$, Nassir Navab ${ }^{2}$, and Dorin Comaniciu ${ }^{1}$ \\ ${ }^{1}$ Integrated Data Systems, Siemens Corporate Research, Princeton, USA \\ ${ }^{2}$ Computer Aided Medical Procedures, Technical University Munich, Germany \\ ${ }^{3}$ Department of Radiology, Medical University of South Carolina, Charleston, USA \\ ${ }^{4}$ Siemens Medical Solutions, Computed Tomography, Forchheim, Germany \\ razvan_ionasec.ext@siemens.com
}

\begin{abstract}
Aortic valve disease is an important cardio-vascular disorder, which affects $2.5 \%$ of the global population and often requires elaborate clinical management. Experts agree that visual and quantitative evaluation of the valve, crucial throughout the clinical workflow, is currently limited to 2D imaging which can potentially yield inaccurate measurements. In this paper, we propose a novel approach for morphological and functional quantification of the aortic valve based on a $4 \mathrm{D}$ model estimated from computed tomography data. A physiological model of the aortic valve, capable to express large shape variations, is generated using parametric splines together with anatomically-driven topological and geometrical constraints. Recent advances in discriminative learning and incremental searching methods allow rapid estimation of the model parameters from 4D Cardiac CT specifically for each patient. The proposed approach enables precise valve evaluation with model-based dynamic measurements and advanced visualization. Extensive experiments and initial clinical validation demonstrate the efficiency and accuracy of the proposed approach. To the best of our knowledge this is the first time such a patient specific $4 \mathrm{D}$ aortic valve model is proposed.
\end{abstract}

\section{Introduction}

Aortic valve disease represents the most common valvular disease in developed countries [1], and shows the second highest incidence among congenital valvular defects [2]. Although, aortic root preserving surgery 3] along with minimally invasive procedures are emerging, the management of patient with valvular heart disease (VHD) has remained challenging. Precise knowledge and reliable display of the four-dimensional valve characteristics are requested by clinicians.

To date, most data on geometry and dynamics were obtained by experimental studies on explanted valves or using animal models 4, with small numbers of

\footnotetext{
* This work was initiated as a joint diploma thesis between Friedrich-AlexanderUniversity of Erlangen-Nuremberg and Siemens Corporate Research. We acknowledge the advice of Prof. Joachim Hornegger and Dr. Martin Huber.

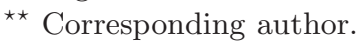

D. Metaxas et al. (Eds.): MICCAI 2008, Part I, LNCS 5241, pp. 686-694 2008.

(C) Springer-Verlag Berlin Heidelberg 2008 


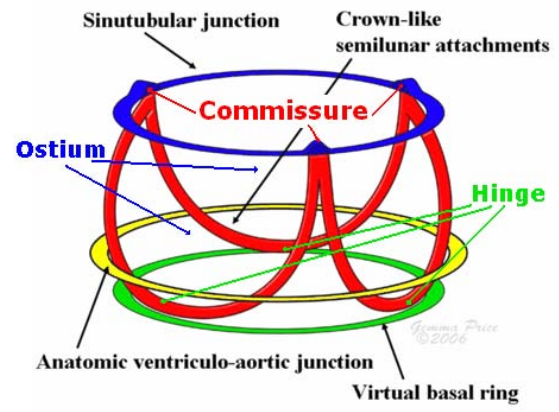

(a)

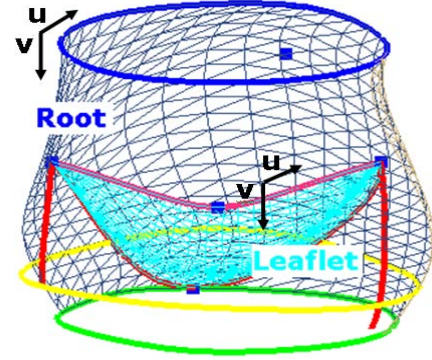

(b)

Fig. 1. (a) A generic aortic valve model in perspective view. Reproduced with permission of the author and the European Association for Cardio-Thoracic Surgery from: Anderson RH (doi:10.1510/mmcts.2006.002527). (b) The proposed aortic valve model in perspective view, where $u$ and $v$ are the circumferential and height parametric direction, respectively, of the root and leaflets.

cases across publications. Standard dimensions of the aortic root are given in literature, though considerable variations in the geometry are recognized [5]. Quantitative and visual evaluation methods are limited to $2 \mathrm{D}$ images and are potentially affected by measurement inaccuracies [6.

Computed tomography is a rapidly evolving modality for non-invasive imaging of the heart which enables dynamic four-dimensional evaluation of cardiac structures throughout the cardiac cycle. However, quantification of the aortic valve is impaired by the need to translate the four-dimensional data set into a set of $2 \mathrm{D}$ planes in order to obtain measurements. This gives potentially erroneous results for the curved anatomy of the basal aorta. A four dimensional model derived from CT data sets offers the unique possibility to non-invasively visualize and quantify the dynamics of the human aortic root and leaflets in healthy and diseased valves.

Existent valve models presented in the literature [7/8, are either used for hemodynamic studies or for analysis of various prosthetic valves. Although some of them are generated from volumetric data, these models are generic and obviously not applicable for the evaluation of individual patients.

In this paper we introduce a new modeling framework for the aortic valve from 4D cardiac CT data. A dynamic model of the valve is constructed from anatomic structures together with physiology driven geometrical and topological constraints (Section 2.1). The patient specific parameters of the model are estimated from CT data by combining learning-based technologies into a three-stage, coarse-to-fine parameter estimation algorithm (Section 2.2): landmark detection, full model fitting and model dynamics estimation.

The estimated model enables for the first time precise morphological and functional quantification as well as enhanced visualization of the aortic valve. This novel model-based evaluation paradigm has the potential to significantly advance the management of valvular heart disease. 
Extensive experiments on 37 patients with various valvular disorders demonstrate the accuracy and speed of the proposed model estimation algorithm (Section 3.1). Initial clinical validation on various healthy and pathological valves shows a strong correlation among a proposed set of model-based measurements, manually performed measurements and previously reported aortic valve dimensions (Section 3.2).

\section{Physiological Valve Modeling}

The morphology and function of the aortic valve is very complex, which is underlined by the lack of consensus regarding its optimal physiological description [9]. The central anatomical structures of the aortic valve are the leaflets and the root. Its function is to regulate the blood flow between the left ventricle and aorta.

\subsection{Model Representation}

We propose a physiology driven parametric 4D model capable to express a large spectrum of morphological and pathological variations of the aortic valve. A set of well-defined landmarks, which includes hinges, commissures, leaflet tips and coronary ostia, describe key anatomical locations of the valve (see Fig. 1(a)]. The aortic root and leaflets form the central anatomic structures and their geometries are represented by Non uniform rational B-splines (NURBS), which is the de facto standard in computational modeling. These components together with topological and geometrical constraints define a physiologically compliant model of the aortic valve, capable to implicitly handle bicuspid malformations.

The aortic root connects the left ventricular outflow tract to the ascending aorta and provides the supporting structures for the leaflets. This is represented by a NURBS surface $C^{\text {root }}(u, v)$ closed in the $u$ parametric direction and can be imagined as a deformed cylinder constrained by the hinge, commissure and ostia points:

$$
\begin{aligned}
& \underbrace{C^{\text {root }}(u, v)}_{u, v \in[0,1]}=\frac{\sum_{i=0}^{n} \sum_{j=0}^{m} N_{i, d}(u) N_{j, e}(v) w_{i, j} \boldsymbol{P}_{\boldsymbol{i}, \boldsymbol{j}}^{\text {root }}}{\sum_{i=0}^{n} \sum_{j=0}^{m} N_{i, d}(u) N_{j, e}(v) w_{i, j}} \\
& \boldsymbol{P}_{l, \boldsymbol{j}}^{\text {root }}=\boldsymbol{P}_{\boldsymbol{l}-\boldsymbol{n}, \boldsymbol{j}}^{\text {root }}, 0 \leq l \leq d, 0 \leq j \leq m
\end{aligned}
$$$$
C^{\text {root }}\left(u_{k}^{h}, v_{k}^{h}\right)=\boldsymbol{L}_{\boldsymbol{k}}^{h}, 0 \leq k<3
$$$$
C^{\text {root }}\left(u_{k}^{c}, v_{k}^{c}\right)=\boldsymbol{L}_{k}^{c}, 0 \leq k<3
$$$$
C^{\text {root }}\left(u_{k}^{o}, v_{k}^{o}\right)=\boldsymbol{L}_{k}^{o}, 0 \leq k<2
$$

where $\boldsymbol{P}_{\boldsymbol{i}, \boldsymbol{j}}^{\text {root }}$ are the control points, $w_{i, j}$ are the corresponding weights, $N_{i, d}(u)$ and $N_{j, e}(u)$ are the $d^{t h}$ and $e^{t h}$ degree B-splines basis functions defined on the non-periodic knot vector $U$ and $V$, respectively. The root surface $C^{\text {root }}$ passes through the hinges $\left(\boldsymbol{L}_{\boldsymbol{h}}^{\boldsymbol{r}}\right)$, commissures $\left(\boldsymbol{L}_{\boldsymbol{c}}^{\boldsymbol{r}}\right)$ and ostia $\left(\boldsymbol{L}_{\boldsymbol{o}}^{\boldsymbol{r}}\right)$ landmarks at parametric location $\left(u_{k}^{h}, v_{k}^{h}\right),\left(u_{k}^{c}, v_{k}^{c}\right)$ and $\left(u_{k}^{o}, v_{k}^{o}\right)$, respectively. A comprehensive description of NURBS is given in [10].

The three valvular leaflets, expressed as NURBS paraboloids, are fixed to the root on an attachment crown delineated by the hinges and commissures, while 
the remaining free edge of the leaflets is constrained by the corresponding tip point. These open and close during the cardiac cycle allowing one way blood flow during systole, from the left ventricle to the aorta:

$$
\begin{aligned}
& \sum_{i=0}^{n} \sum_{j=0}^{m} N_{i, d}(u) N_{j, e}(v) w_{i, j} P_{i, j}^{\text {leaf }}{ }_{i, j} \\
& \underbrace{C^{\text {leafl }}(u, v)}=\frac{\sum_{i=0}{ }_{j=0}^{n}}{\sum^{n} \sum^{m} N_{i, d}(u) N_{j, e}(v) w_{i, j}}\left(u_{l}^{h}, v_{l}^{h}\right)=\boldsymbol{L}_{l}^{h} \\
& \sum_{u, v \in[0,1]} \sum_{i=0} N_{i, d}(u) N_{j, e}(v) w_{i, j} \quad C^{\text {leaf }}{ }^{l}\left(u_{l}^{t}, v_{l}^{t}\right)=\boldsymbol{L}_{l}^{t} \\
& \boldsymbol{P}^{\text {leaf }}{ }_{0,0}^{l}=\boldsymbol{P}^{\text {leaf }}{ }_{\mathbf{0}, j}^{l}=\boldsymbol{L}_{l}^{c}, 0 \leq j \leq m \quad C^{\text {leafl }}(u, 0)=C^{\text {root }}\left(u_{k}^{l}, v_{k}^{l}\right) \\
& \boldsymbol{P}^{\text {leaf }}{ }_{n, 0}^{l}=\boldsymbol{P}_{n, j}^{\text {leaf }}{ }_{n, j}^{l}=\boldsymbol{L}_{l+1}^{c}, 0 \leq j \leq m
\end{aligned}
$$

where $C^{\text {leaf }}$ stands for the $l^{\text {th }}$ leaflet surface and $\boldsymbol{P}^{\text {leaf }}{ }_{\boldsymbol{i}, \boldsymbol{j}}^{\boldsymbol{l}}, w_{i, j}, N_{i, d}(u)$ and $N_{j, e}(u)$ are defined analogous to equation (1). The surface converges into the adjacent commissures $\left(\boldsymbol{L}_{\boldsymbol{l}}^{\boldsymbol{c}}\right.$ and $\left.\boldsymbol{L}_{\boldsymbol{l}+\mathbf{1}}^{c}\right)$, passes through the corresponding hinge $\left(\boldsymbol{L}_{\boldsymbol{l}}^{\boldsymbol{h}}\right)$ and tip $\left(\boldsymbol{L}_{\boldsymbol{l}}^{\boldsymbol{t}}\right)$, and the $0-i$ socurve lies on the root at parametric locations $\left(u_{k}^{l}, v_{k}^{l}\right)$.

It is straightforward within the NURBS framework to extend the above presented 3D model to a dynamic model (4D) using the tensor product, which introduces a temporal parametric direction $t$ to the model representation 10 .

\subsection{Model Estimation}

The parameters of the valve model proposed in section 2.1 are estimated for each patient from $4 \mathrm{D}$ cardiac CT data. A specific instance of the model is exactly determined by the landmarks and NURBS control points in a four-dimensional Euclidean space $(3 \mathrm{D}+$ time $)$, which cumulates into $3 T(11+300)$ parameter 1 . Due the high dimension of the parameter vector, direct estimation in the original space is very difficult. We propose a three-step approach to estimate the parameters of the dynamic valve model, which are: landmarks detection, full model fitting and model dynamics estimation.

Landmark Detection. Recent advances in discriminative learning and incremental searching techniques are applied to automatically determine the landmarks locations $\left(\boldsymbol{L}_{\boldsymbol{h}}^{\boldsymbol{r}}, \boldsymbol{L}_{\boldsymbol{c}}^{\boldsymbol{r}}, \boldsymbol{L}_{\boldsymbol{o}}^{\boldsymbol{r}}\right.$ and $\left.\boldsymbol{L}_{\boldsymbol{t}}^{\boldsymbol{r}}\right)$ from an input volume. A training set, which contains positive and negative samples of the landmarks positions, is created from a manually annotated database. We train a discriminative classifier $H(x, y, z)$ based on the Probabilistic Boosting Tree (PBT) [11, which learns the target distribution by exploiting a divide-and-conquer strategy:

$$
p\left(\boldsymbol{L}_{\boldsymbol{i}} \mid x_{s}, y_{s}, z_{s}\right)=H_{i}\left(x_{s}, y_{s}, z_{s}\right), x_{s}, y_{s}, z_{s} \in D_{i}
$$

\footnotetext{
${ }^{1} T$ represents the number of discrete samples in the time dimension (10 for a regular $4 \mathrm{D}$ cardiac CT acquisitions), 11 and 300 the number of landmarks and control points, respectively.
} 


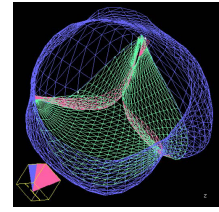

(a) End-Diastole

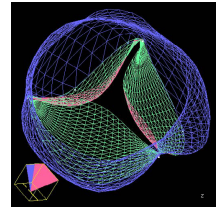

(b)

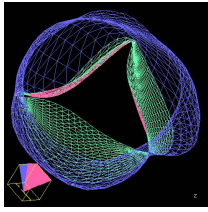

(c)

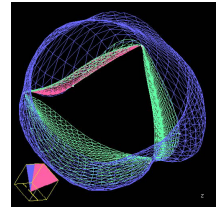

(d) End-Systole

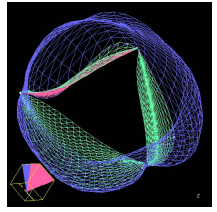

(e)

Fig. 2. (a-e) Five frames of the dynamic valve model obtained from a CT sequence

where $p\left(\boldsymbol{L}_{\boldsymbol{i}} \mid x_{s}, y_{s}, z_{s}\right)$ is the probability of landmark $\boldsymbol{L}_{\boldsymbol{i}}$ at location $\left(x_{s}, y_{s}, z_{s}\right)$, $H_{i}$ the strong classifier trained for $\boldsymbol{L}_{\boldsymbol{i}}$ and $D_{i}$ the search domain obtain from the training set. The detection is performed in a coarse-to-fine manner as well as in incrementally increasing parameter spaces similar to the marginal space learning (MSL) 12 concept.

A thin-plate-spline (TPS) transformation 1314 is computed from the detected set of corresponding points, $K=\left\{\left(\boldsymbol{L}_{\boldsymbol{i}}^{\boldsymbol{m}}, \boldsymbol{L}_{\boldsymbol{i}}^{\boldsymbol{I}}\right), \boldsymbol{L}_{\boldsymbol{i}}^{\boldsymbol{m}} \in M, \boldsymbol{L}_{\boldsymbol{i}}^{\boldsymbol{I}} \in I, 0<i \leq N\right\}$, which maps each control point of previously computed mean shape $M$ at the corresponding location in the image $I$ and provides an initial model estimation.

Full Model Fitting. The initial estimation obtained through landmark detection and TPS transformation provides a quite accurate global fitting of the model, however it requires further local processing for precise object delineation. A boundary detector is trained using the PBT algorithm in combination with steerable features, proposed in [12]. This is applied locally at a set of discrete boundary locations and is used to evolve the shape towards high probability responses of the boundary detector. The final estimation is obtained by fitting the parametric model to the refined samples by solving a linear least squares problem [15].

Model Dynamics Estimation. The estimation of the dynamic valve model follows a physiology-driven strategy and is more accurate and efficient compared to a sequential computation of the input sequence. Parameter estimation is initially performed for the dominant shapes observed in the end-diastolic (valve is completely closed) and end-systolic (valve is completely opened) cardiac phases, according to the algorithm described above. The estimation for the remaining frames exploits a prior model constructed as a linear combination of the two reference shapes, leading to a significant performance boost. Fig. 2 illustrates the dynamic model of the aortic valve estimated from a CT cardiac sequence.

\section{Results}

\subsection{Results on Valve Model Estimation}

We demonstrate the performance of the proposed algorithm on $374 \mathrm{D}$ cardiac CT data set, which consist of $364 \mathrm{CT}$ volumes. The scans are acquired from 


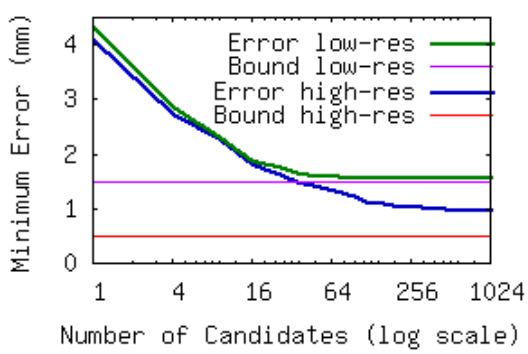

(a)

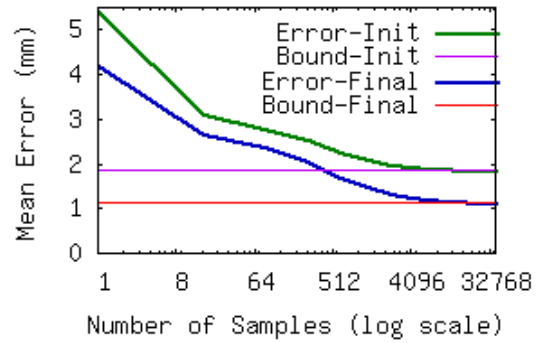

(b)

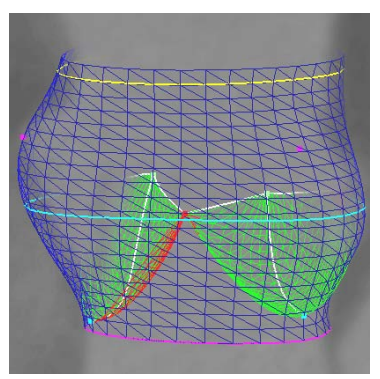

(c)

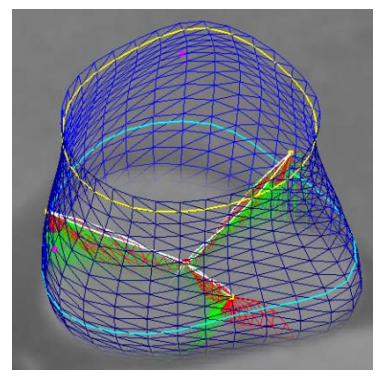

(d)

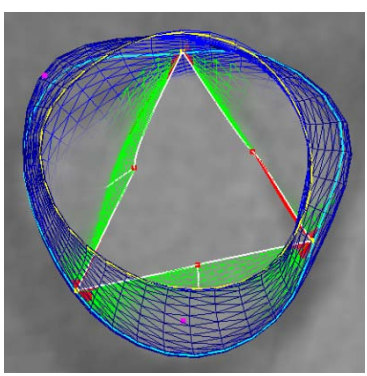

(e)

Fig. 3. (a) The detection error for the landmarks with respect to the cardinality of the candidates set. (b) The error curve for model estimation with respect to the number of samples. (c-e) Model estimation results in fused visualization.

different patients with various cardiovascular diseases using heterogeneous protocols, resulting in significant resolution and capture range variation (80 to 350 slices with sizes from $512 \times 512$ to $153 \times 153$ pixels and resolution from $0.28 \mathrm{~mm}$ to $2.0 \mathrm{~mm}$ ). Each volume in the data set is associated to an annotation, which was manually generated and is considered to be the ground truth. Three-fold cross validation is performed in order to evaluate our algorithm.

Performance of Landmark Detection. The landmark detection is evaluated by the Euclidean distance between the ground truth and detected positions. Initially, the detection is performed in low-resolution images and generates a set of position hypotheses for each landmark. Fig. 3(a) shows the error of the most accurate hypothesis, averaged over all landmarks, with respect to the cardinality of the hypotheses set. An optimal trade-of between speed and accuracy is achieved by keeping 100 candidates with an error of $1.59 \mathrm{~mm}$. Detection is refined in high resolution images $(1 \mathrm{~mm})$, which enables $10 \%$ increase in accuracy for $30 \%$ hypothesis. Averaging over the candidates set results in the final detection error of $2.28 \mathrm{~mm}$.

Performance of Model Estimation. The model estimation accuracy is evaluated by the point-to-mesh measurement, which computes the average distance between sample pairs of the detected and ground-truth model. The accuracy of the initial model estimation relative to the rendering and computation resolution 
used within the system (2500 samples) is on average $2.00 \mathrm{~mm}$. The model refinement improves the estimation accuracy by nearly $40 \%$ (see Fig. 3(b)], equivalent to an error of $1.33 \mathrm{~mm}$. The computation time of the proposed method was evaluated on a standard desktop machine (3.0 GHz CPU, 2.0 GB RAM). The estimation of the full dynamic valve model from a regular CT sequence (10 volumes), is computed in 21.3 seconds with $70 \%$ of the time required for the landmark detection.

\subsection{Results on Clinical Valve Evaluation}

We demonstrate the quantitative and visual capabilities and underline the performance of the proposed method by comparing a set of morphological and dynamic model-based measurements to expert measurements and literature reported valve dimensions. Evaluation is performed on CT images of healthy, stenotic, dilated aorta and bicuspid valves, while the ground truth is provided by measurements manually performed by a radiologist with five years of experience in cardiovascular imaging. Table 1 summarizes the evaluation results and demonstrates the precision of the proposed model-based quantification method.

Table 1. Model-based quantification error with respect to expert measurements

\begin{tabular}{|c|c|c|c|c|c|c|c|}
\hline & VAJ $(\mathrm{cm})$ & SV $(\mathrm{cm})$ & STJ $(\mathrm{cm})$ & AVA $\left(\mathrm{cm}^{2}\right)$ & LCT $(\mathrm{mm})$ & RCT $(\mathrm{mm})$ & NCT $(\mathrm{mm})$ \\
\hline \hline Mean & 0.137 & 0.166 & 0.098 & 0.120 & 2.211 & 1.951 & 2.352 \\
\hline STD & 0.017 & 0.043 & 0.029 & 0.380 & 0.866 & 0.936 & 1.162 \\
\hline
\end{tabular}

The root diameter, important in surgical treatment of dilated and stiff anatomies 44 is computed at three levels: ventricular-arterial junction (VAJ), sinus of valsalva (SV) and sinotubular junction (SJ). Severity assessment in patients with degenerative aortic stenosis is supported through the aortic valve area (AVA) measurement [6]. The mean AVA derived from the model was 3.74 $1.34 \mathrm{~cm}^{2}$, correlation with respect to manual planimetry $r=0.963$ and $p<$ 0.0001 , and Bland-Altman systematic bias $0.12 \pm 0.38 \mathrm{~cm}^{2}$. Left-coronary tip (LCT), non-coronary tip (NCT) and right-coronary tip (RCT) orthogonal excursion is proposed for the evaluation of valve's function and motion characteristic after surgery. Measurements variation in healthy and diseased valves is illustrated in Fig. 4 .

Fused visualization of direct volume rendering (DVR) of the 4D CT dataset and the estimated model provides further anatomical insight. Advanced techniques [16] enable visualization via post color-attenuated transfer functions [17] of the aortic lumen from CTA and integration of the valve model geometry into the DVR. Combination of CT data and model geometry can be directly used for visual validation of the estimated parameters with respect to the anatomy and visual quantification of pathological valves. For real-time $4 \mathrm{D}$ rendering of the sequence we use GPU Raycasting to efficiently stream the volume and geometry data to the graphics card. The combination of these techniques is capable of rendering high quality images, at interactive frame rates (Fig. 4(a), 4(b)) . 


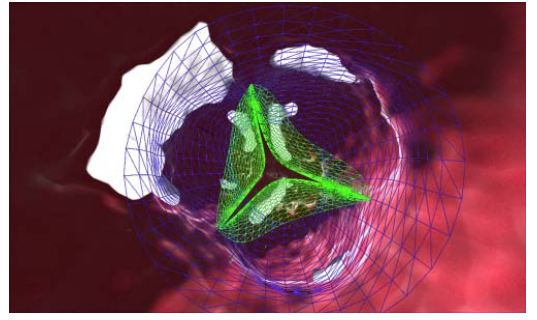

(a)

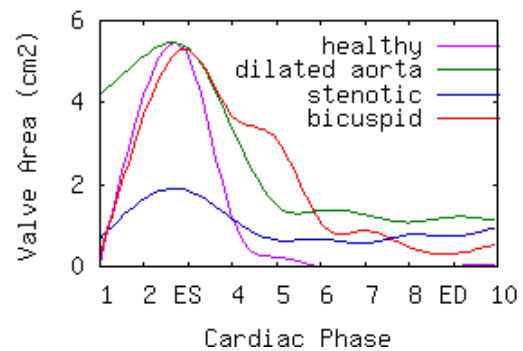

(c)

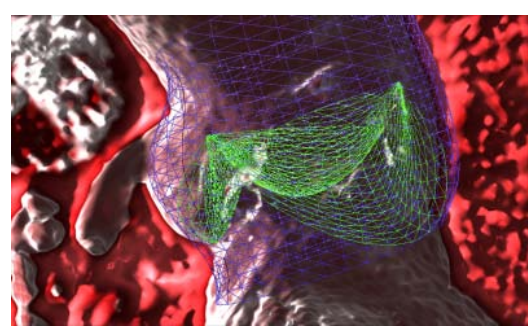

(b)

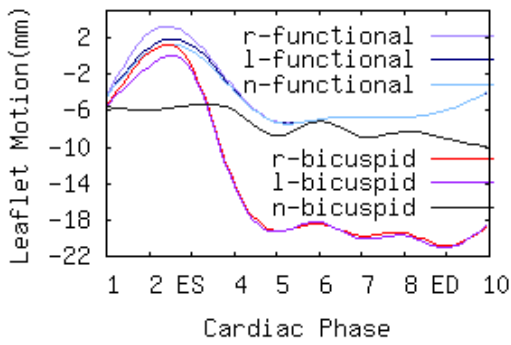

(d)

Fig. 4. Advanced visualization of (a) calcified aortic valve in endoscopic view and (b) bicuspid valve in long axis view. (c,d) Valve area and leaflet motion variation in different pathologies.

\section{Discussion}

This paper proposes a novel quantitative and visual evaluation approach of the aortic valve, based on a dynamic model estimated from $4 \mathrm{D}$ CT sequences. A robust and computationally efficient algorithm, which combines learning-based technologies into a coarse-to-fine approach, was proposed for estimating a patient specific valve model from imaging data. Automatic model-based measurements provide a significant advance in morphological and functional clinical evaluation of the aortic valve, currently limited to 2D imaging methods, operator-dependent and potentially inaccurate measurements. Future research will include high-level information provided by soft tissue composition and mechanical constrains, as well as investigations for other modalities (e.g. Ultrasound and MRI).

\section{References}

1. Nkomo, V., Gardin, J., et al.: Burden of valvular heart diseases: a population-based study. Lancet. 368(10), 1005-1011 (2006)

2. Hoffman, J., Kaplan, S.: The incidence of congenital heart disease. J. Am. Coll. Cardiol. 39(12), 1890-1900 (2002)

3. Yacoub, M.: Late results of a valve-preserving operation in patients with aneurysms of the ascending aorta and root. J. Thorac. Cardiovasc. Surg. 115, 1080-1090 (1998)

4. Dagum, P., Green, G., et al.: Deformational dynamics of the aortic root: modes and physiologic determinants. J. Thorac. Cardiovasc. Surg. 100(19), II54-62 (1999) 
5. Labrosse, M.: Geometric modeling of functional trileaflet aortic valves: development and clinical applications. J. Biomech. 39(14), 2665-2672 (2006)

6. Vahanian, A., Baumgartner, H., et al.: Guidelines on the management of valvular heart disease: The task force on the management of valvular heart disease of the european society of cardiology. European heart journal 28(2), 230-268 (2007)

7. Peskin, C.S., McQueen, D.M.: Fluid dynamics of the heart and its valves. In: Othmer, H.G., Adler, F.R., Lewis, M.A., Dallon, J.C. (eds.) Case Studies in Mathematical Modeling: Ecology, Physiology, and Cell Biology, pp. 309-337. Prentice-Hall, Englewood Cliffs (1996)

8. De Hart, J., Peters, G., et al.: A three-dimensional computational analysis of fluidstructure interaction in the aortic valve. J. Biomechanics 36(1), 103-110 (2002)

9. Anderson, R.: The surgical anatomy of the aortic root. Multimedia Manual of Cardiothoracic Surgery (MMCTS) (2006)doi:10.1510/mmcts.2006.002527

10. Piegl, L., Tiller, W.: The NURBS book. Springer, London (1995)

11. Tu, Z.: Probabilistic boosting-tree: Learning discriminative methods for classification, recognition, and clustering. In: ICCV 2005, pp. 1589-1596 (2005)

12. Zheng, Y., Barbu, A., et al.: Fast automatic heart chamber segmentation from 3D ct data using marginal space learning and steerable features. In: ICCV (2007)

13. Duchon, J.: Interpolation des fonctions de deux variables suivant le principe de la flexion des plaques minces. RAIRO Analyse Numerique 10, 5-12 (1976)

14. Bookstein, F.L.: Principal warps: Thin-plate splines and the decomposition of deformations. IEEE PAMI 11(6), 567-585 (1989)

15. DeBoor, H.: A Practical Guide to Splines. Springer, New York (1978)

16. Scharsach, H., Hadwiger, M., Neubauer, A., Wolfsberger, S., Buhler, K.: Perspective Isosurface and Direct Volume Rendering for Virtual Endoscopy Applications. In: Proceedings of Eurovis/IEEE-VGTC Symposium on Visualization 2006, pp. 315-322 (2006)

17. Zhang, Q., Eagleson, R., Peters, T.: Rapid Voxel Classification Methodology for Interactive 3D Medical Image Visualization. In: Ayache, N., Ourselin, S., Maeder, A. (eds.) MICCAI 2007, Part II. LNCS, vol. 4792, pp. 86-93. Springer, Heidelberg (2007) 